\title{
Robustness against Fading Fluctuation in Hermite-Symmetric Subcarrier Coding for OFDM Systems
}

\author{
Akira Toda, Fumihito Sasamori, Osamu Takyu, and Shiro Handa \\ Shinshu University, 4-17-1 Wakasato, Nagano-shi, Nagano 380-8553, Japan \\ E-mail: 13tm233a@shinshu-u.ac.jp
}

\begin{abstract}
An Hermite-symmetric subcarrier coding for OFDM systems (HC-OFDM) enables dual channel transmission over individual IQ wireless channels with higher power efficiency than a simple repetition coding (RC-OFDM). We theoretically proved the advantage of the HC-OFDM systems assuming an ideal coherent detection. But, in practice, the dual channel transmission needs precise phase compensation to separate each channel. In this paper, we evaluate the robustness against fading fluctuation in the HC-OFDM systems.
\end{abstract}

\section{Keywords - Hermite-Symmetric Subcarrier Coding, OFDM, Phase compensation}

\section{INTRODUCTION}

In recent years, wireless communications with high quality and high speed are greatly desired. Orthogonal Frequency Division Multiplexing (OFDM) is well-known as the technology to meet the requirements. We have focused on the OFDM systems with a repetition coding on the frequency domain (RC-OFDM) which realize the diversity technique with one pair of transmit/receive antennas [1]. On the other hand, with a trade-off relation ship, there is a disadvantage that the transmission rate is degraded in order to send the same signal. We have proposed Hermite-symmetric subcarrier coded OFDM (HC-OFDM) systems to keep the transmission rate, and theoretically proved the advantage of the HC-OFDM systems assuming an ideal coherent detection [2]. The purpose of study is to consider a phase compensation on signal processing and to prove the advantage practically.

\section{System MODEL}

Figure 1 (a) shows a subcarrier coding scheme of the RCOFDM systems, where $N_{c}$ is the number of subcarriers. At a transmitter, a digital-modulated symbol (PSK or QAM) $D_{i}$ $\left(i=1, \cdots, N_{c} / 2\right)$ is duplicated with equal frequency spacing in the subcarrier mapping. At a receiver, the two symbols are combined with maximum ratio combining (MRC). Figure 1 (b) shows a subcarrier coding scheme of the HC-OFDM systems. The two symbols have the Hermite-symmetry, then the imaginary part (Q-channel) of the generated OFDM signal can be forced to be zero. This is based on the fact that the Fourier transform of real-valued input without imaginary part becomes Hermite-symmetric. Therefore, another data can be simultaneously transmitted in Q-channel, which we call 'dual channel transmission.'

We theoretically proved the advantage of the HC-OFDM systems assuming an ideal coherent detection [2]. But, in practice, the dual channel transmission needs a precise phase compensation to separate each channel. In this paper, we evaluate the performance including the phase compensation on signal processing.

Figure 2 shows a system model of the phase compensation. A pilot signal is transmitted to calculate a channel state information (CSI) at each subcarrier. The multipath fading can be considered as the discrete-time convolution on the OFDM signal processing. Therefore, after the received OFDM signal is converted to the frequency domain and simply divided by the CSI at each subcarrier, IQ imbalance by multipath fading can be compensated by the signal processing.

\section{PERFormance EVAluAtion}

Table I shows the simulation specifications. The RC-OFDM needs higher-level digital modulation to compensate the degradation of the transmission rate. Unlike the RC-OFDM, the HC-OFDM can use the same digital modulation of the general OFDM because of the dual channel transmission in case of the same rate condition. On the other hand, the signal power per

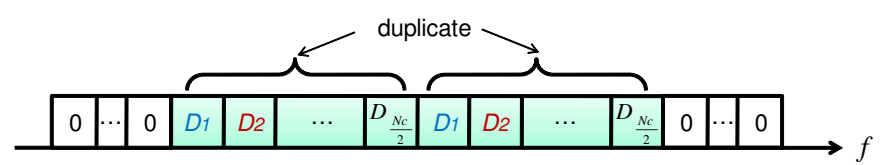

(a) RC-OFDM

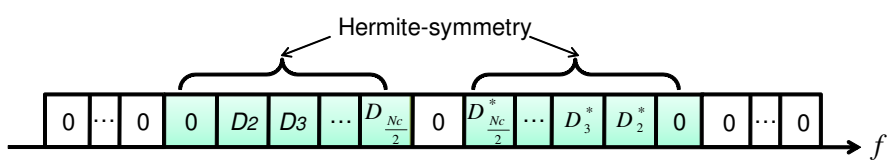

(b) HC-OFDM

Fig. 1. Sucarrier coding scheme

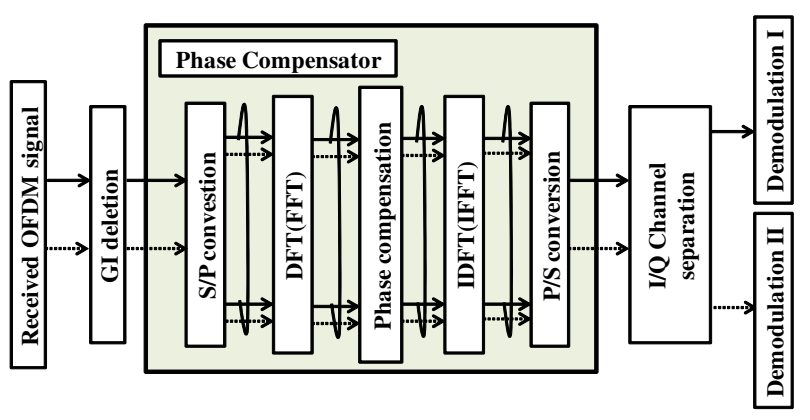

Fig. 2. A concept of Phase Compensation 
channel in the HC-OFDM should be reduced by half in order to keep the same carrier power condition [2].

In this section, we evaluate average bit error rate (BER) performances comparing the general OFDM, the RC-OFDM and the HC-OFDM in the equal condition.

\section{A. Performance evaluation in quasi-static fading environment}

Figure 3 shows an average BER performance without fading fluctuation in the time domain, that is, in the case of Doppler frequency normalized by OFDM symbol duration $f_{D} T_{s}=$ 0.0. It is found from the figure that the phase compensation works well in the dual channel transmission of the HC-OFDM. Moreover, the HC-OFDM and the RC-OFDM can attain better performance than the general OFDM owing to the frequency diversity gain. The HC-OFDM outperforms the RC-OFDM because of the power efficiency in the digital modulation.

When once the CSI at each subcarrier is estimated, we can continue to utilize it without renewal in the quasi-static fading environment. But, considering wireless mobile channel scenarios, the CSI estimation error caused by fading fluctuation in the time domain should be considered. Therefore, we will compare the robustness between the HC-OFDM and the RCOFDM in the next subsection.

\section{B. Performance evaluation with fading fluctuation in time domain}

Figure 4 shows an average BER performance of the HCOFDM and the RC-OFDM with fading fluctuation in the case of $f_{D} T_{s}=0.005$ and 0.02 . The Doppler fluctuation deteriorates the orthogonality of IQ wireless channels and causes inter carrier interference (ICI), then the performance is degraded by the CSI estimation error. In the case of fast Doppler frequency like $f_{D} T_{s}=0.02$, irreducible bit error (bit error floor) occurs in both OFDM systems. But, it is found from the figure that the HC-OFDM outperforms the RCOFDM even if the value of $f_{D} T_{s}$ is increased. Since the QPSK in the HC-OFDM have the advantage of power efficiency against the 16QAM in the RC-OFDM, the interference power in the HC-OFDM becomes relatively low. Therefore, the HC-OFDM can reduce the performance degradation by the

TABLE I

SiMULATION SPECIFICATIONS.

\begin{tabular}{c|c}
\hline Modulation & $\begin{array}{c}\text { OFDM (QPSK) } \\
\text { RC-OFDM }(16 Q A M) \\
\text { HC-OFDM }(\text { QPSK } \times 2 \mathrm{ch})\end{array}$ \\
\hline Number of FFT points & 64 \\
\hline Number of Subcarriers & 48 \\
\hline Guard Interval & 16 \\
\hline Wireless Channel & Rayleigh + AWGN \\
\hline Normalized RMS Delay Spread & 0.005 \\
\hline $\begin{array}{c}\text { Normalized Maximum } \\
\text { Doppler Frequency } \\
\left(f_{D} T_{s}\right)\end{array}$ & $0.0,0.005,0.02$ \\
\hline
\end{tabular}

Doppler frequency, that is, the HC-OFDM has the robustness against the fading fluctuation in time domain as compared with the RC-OFDM.

\section{CONCLUSION}

In this paper, we evaluated the superiority of the HC-OFDM over the RC-OFDM. At first, we explained the practical scheme of the phase compensation and confirmed that the IQ wireless channels can be separated at the dual channel transmission. Even if assuming the fading fluctuation scenarios, we confirmed the robustness and the suppression of bit error floor. As future works, we intend to apply adaptive modulation to the HC-OFDM for the purpose of improving throughput.

\section{ACKNOWLEDGMENT}

This study was supported by JSPS KAKENHI Grant Number 24560448 .

\section{REFERENCES}

[1] F. Sasamori, et al., "Performance Analysis of Repetition Coded OFDM Systems with Diversity Combining and Higher-level Modulation," IEICE Trans. on Communications, vol.E94-B, no.1, Jan. 2011.

[2] F. Sasamori, et al., "Performance Analysis of Hermite-Symmetric Subcarrier Coding for OFDM Systems over Fading Channels," IEICE Trans. on Communications, vol.E95-A, no.12, Dec. 2012.

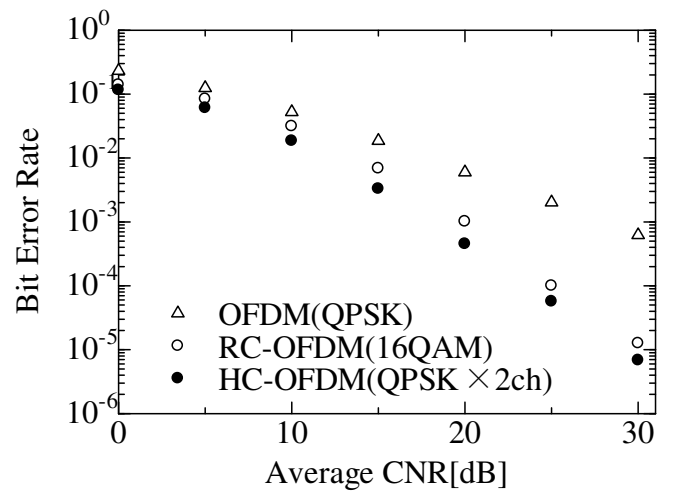

Fig. 3. Average BER performance in quasi-static fading environment

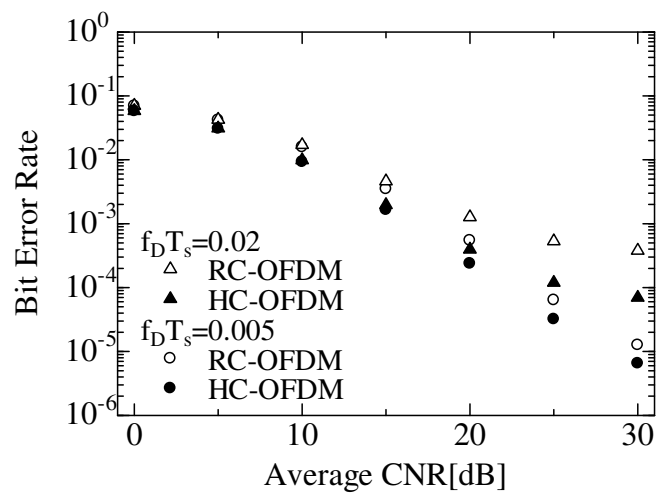

Fig. 4. Average BER performance with fading fluctuation in time domain 